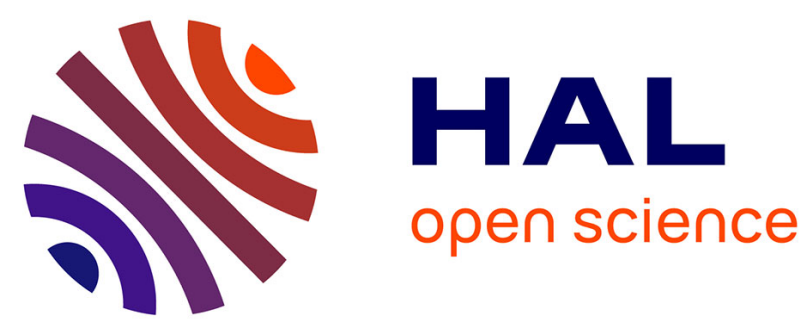

\title{
Le cimetière mérovingien de Saint-Peyre à Félines-Minervois (Hérault)
}

\author{
Louis Méroc, Georges Fouet
}

\section{To cite this version:}

Louis Méroc, Georges Fouet. Le cimetière mérovingien de Saint-Peyre à Félines-Minervois (Hérault). Gallia - Fouilles et monuments archéologiques en France métropolitaine, 1961, 19 (1), pp.191-200. 10.3406/galia.1961.2319 . hal-01926175

\section{HAL Id: hal-01926175 \\ https://hal.science/hal-01926175}

Submitted on 25 Feb 2020

HAL is a multi-disciplinary open access archive for the deposit and dissemination of scientific research documents, whether they are published or not. The documents may come from teaching and research institutions in France or abroad, or from public or private research centers.
L'archive ouverte pluridisciplinaire HAL, est destinée au dépôt et à la diffusion de documents scientifiques de niveau recherche, publiés ou non, émanant des établissements d'enseignement et de recherche français ou étrangers, des laboratoires publics ou privés.

\section{(ㅇ)(1) $\$$}

Distributed under a Creative Commons Attribution - NonCommercial - NoDerivatives $\mid 4.0$ 


\title{
LE CIMETIÈRE MÉROVINGIEN DE SAINT-PEYRE A FÉLINES-MINERVOIS (IIérault)
}

\author{
par M.M. I. Mŕnoc al Gi. Foret
}

Dans le courant du mois de décembre 19:7, à Félines-Minervois (Héranult), Landis qu'ils procédaient au sous-solage de la parcelle 17 au lieu-dit Saint-Peyre (section $\mathrm{B}$ du cadastre de Félines, 1815), en vue d'une replantation de vigne, MM. Pierre Lignières et. Émile Vaissière remarquaient sur la bordure orientale du champ, parmi les affleurements rocheux qu'ils détruisaient, une sépulture formée de dalles disposées en V renversép Les plantations antérieures avaient, sans aucun doute, déjà détruit plusieurs sépultures sur 'e mème site : les travaux de labours viennent en effet de révéler, par places, des dispersions de dalles, ct a deux reprises, des ossements épars accompagnés, dans un cas, d'une boucle (1 et 2, fig. 10). I'autres inhumations subsistent certainement, sous les nouvelles plantations. Les faibles sondages dont nous allons donner l'analyse, fournissent un aperȩu de cet intéressant, quoique modeste, cimetière campagnard aux caractères bien particuliers (fig. 1 et 2 ).

Les neuf tombes reconnues etaient constituées par des coftrages de dalles de calcaire local, construits d'identique façon : une dalle horizontale, de forme trapézoüdale allongée, formait le fond de la fosse. Posées de champ, à la tête el aux pieds, d'autres dalles fermaient, les extrémités du toit en bâtière. I)es superpositions de pierres, obturant les interstices, entouraient le tout. Ies points les plus élevés de ces accumulations de dalles flête des sépultures) se rencontrèrent entre $0 \mathrm{~m}$. 20 ot $0 \mathrm{~m}$. 50 de profondeur sous l'actuelle surface du sol.

(1) Ils demandirent aussitot a leurs collegues toulousains de venir d'urgence verifier l'interèt arehelogigue de

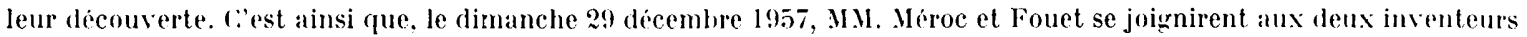
pour finir de dexager et fouiler cette premiere inhumation, et trois autres, presentant les memes caractires, reconnues entre temps. Ia parcelle 17 phantée peu apros, MX. Lignieres et laissieres, poursuivirent en l95s, leurs travaux de sous-solage sur la parcelle no 16 attenante, où ils ne tardirent pas a mettre au jour, non loin des premieres, cinc nou-

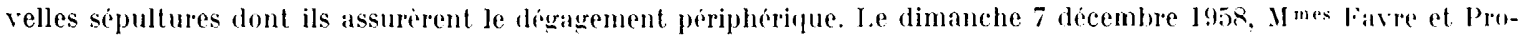
melle, MII. Bousquet. Darolles. Fouet, Jean de lahitte, Meroce Fernand et Alain Promelle, et Tollon. vinrent les aider a relever ef fouiller ces lombes le plus soigneusement possible, arant nivellement du terrain.

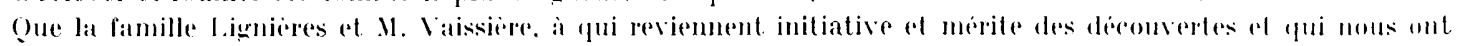
foujours reservi le plus amical aceucil a felines, veullent bien trouver ici lexpression de notre plus vive gratitude. 


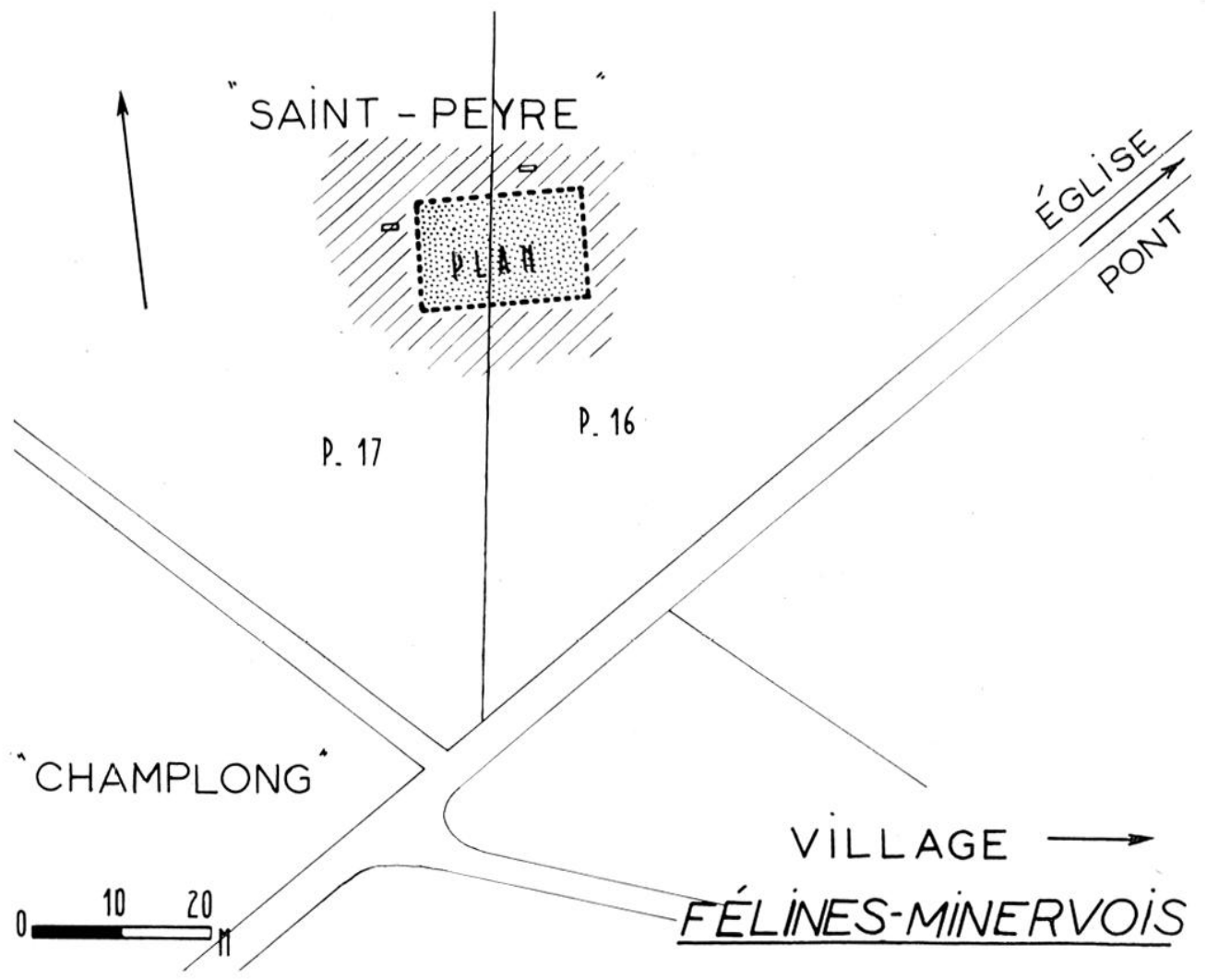

Fig. 1. - Situation_du cimetière de Saint-Peyre.

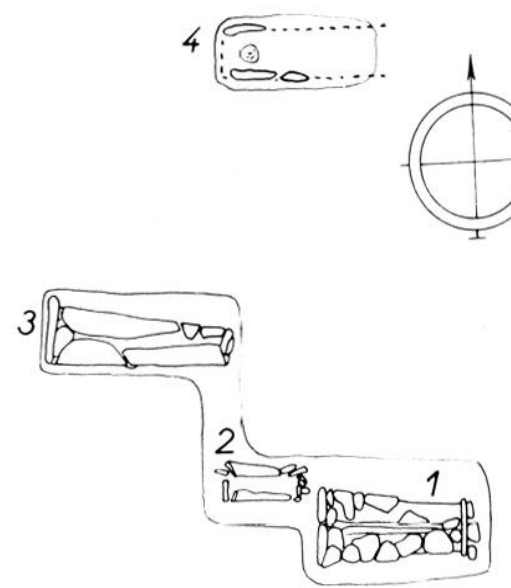

PARCELLE 17

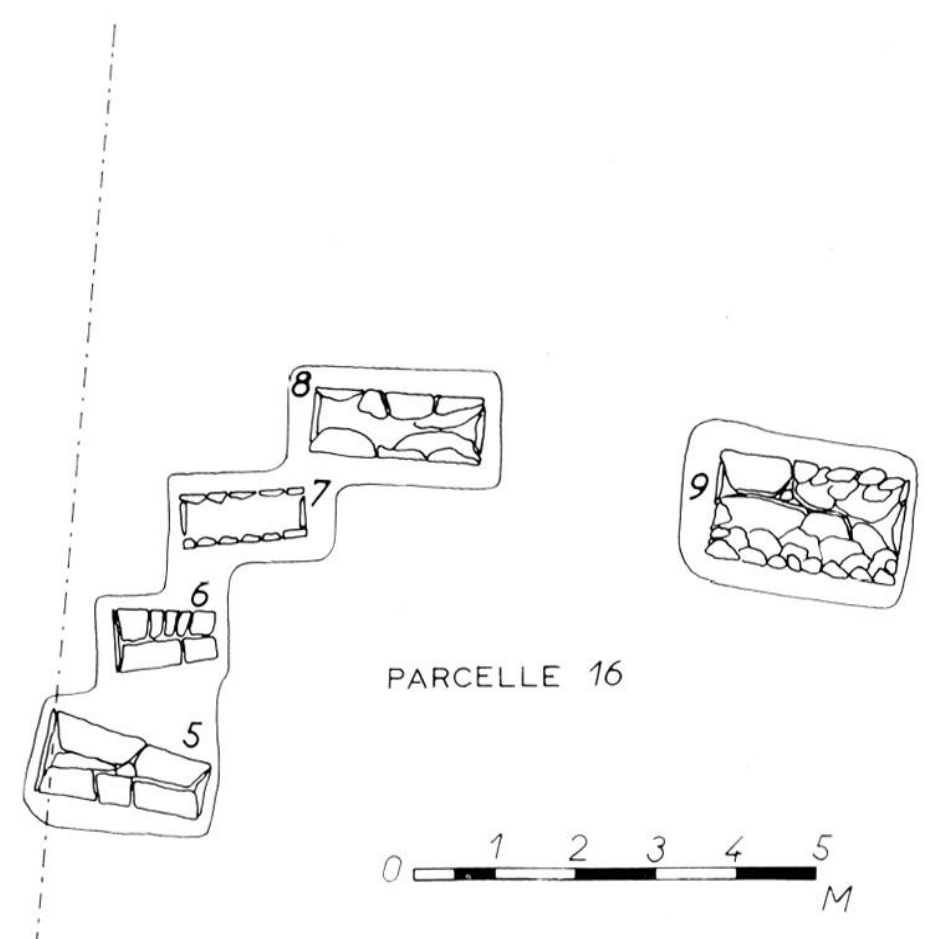

Fig̣. ?. Plan des sondages. 
Tombe 1 (fig. 3). Intacle quoique assez superfiejelle sa hauleur diminuait de la lab alux pieds. de 60 a 40 centimelres. I a dalle de fond mesurait $1 \mathrm{~m}$. 80 de longueur. 1 m. t5 de largeur en tite al

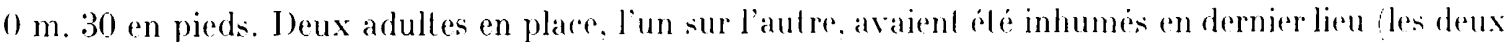
mains repliées sur le bassin pour l'un. les deux mains le long du rorps pour l'aut re postérieurement ¿ 3 occupants antérieurs (adulte, adolescent, enfanl) dont la plupart des ossements araient été regroupés aux deux extrémités el sur les bordures latérales. I ne bonne partie de ces lrois derniers squelettes blait cependant demeurée en place, enrobee dans une couche épaisse de 8 centimelress d'une argile fine claire de consist ance collö̈dale déposée sur la dalle de fond, par les eaux d'infill ration. Au-dessus. les derniers inhumes éaienl parliellemenl recouverts de terre grumeleuse plus foncée. peut-ilre descendue par les inlerstices, conlenant des grains de charbons, quelques petils escarools.

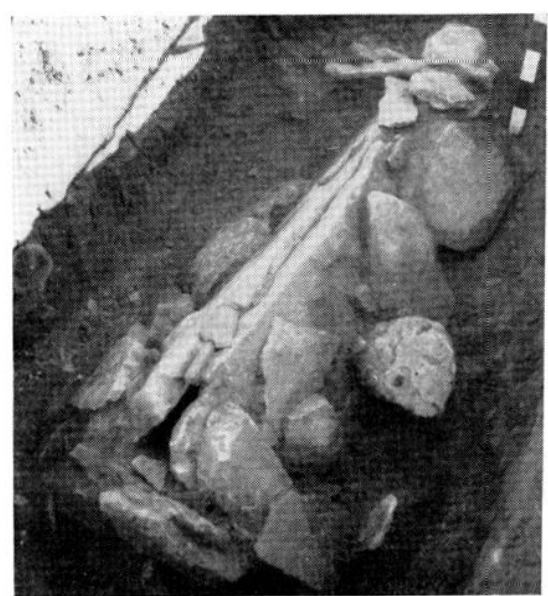

Fifr. 3. Tombe $n^{\circ} 1$, intacte. avint ouverture. Phol" ti. Fonel.

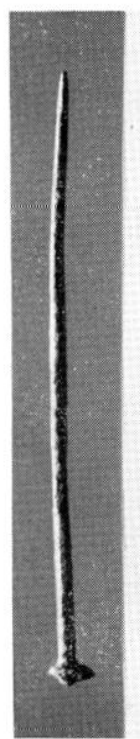

liin. 1.

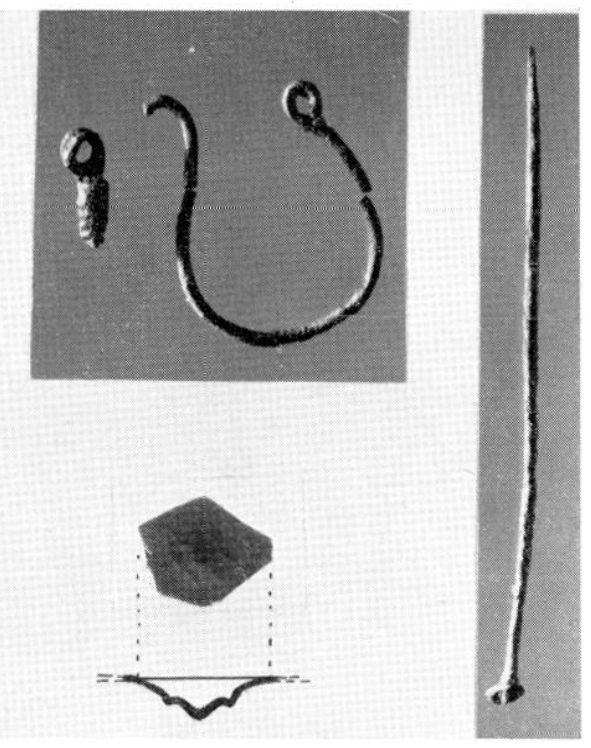

Mobilier de la lomber no 1. le tout an bronze.

réents sans doule. el les pelits objets en bronze de la figure t. dispersés fépingles a une vinglaine de centimetres de l'extrémité ()uest). Ie dégagement du pourlour du coflrage a livé un clon de fer. dans les lerres remuées, a 10 centimetres de l'angle exlérieur sud-list des pieds.

Tombe $\%$. I a partie supérieure de la couverture en bal iere avait été dél ruite par la planlation d'un cep de l'ancienne vigne jusle au centre du coflrage. Ia dalle de fond avail $0 \mathrm{~m}$. 9:3 de longueur pour $0 \mathrm{~m}$. 28 de largeur en lite et $0 \mathrm{~m}$. 2.2 en pieds. I) squelet te du tout jeune enfanl. a dentition de lait, inhumé là. subsistaient seulement une partie de la boîle cranienne et quelques fragments tris endommages des os longs des membres inférieurs deplacés par les racines. I e remplisiage lerrenx renfermait. dans la région des pieds, un menu tesson de polerie noire susceptible de provenir d'une urne romaine lardive.

Tombe 3. Les plantations avaient deplace la moitie de la couverture en loil. Le fond, de $\because$ m. 10 de longueur sur 0 m. $4: 3$ a 0 m. 27 de largeur. étail forme de deux dalles plates, non jointives, oceupant chacune approximativement mi-longueur.

Celle sepulture élait relle d'un adulte aux os assor quiles, mal conserves. In jeune cufant avail eté ajoule quelques annés plus lard et les os lones des membres inférieurs de ladulte araiend

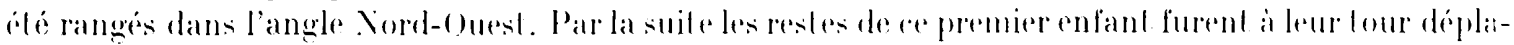

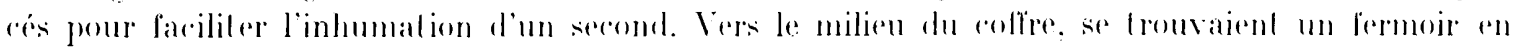



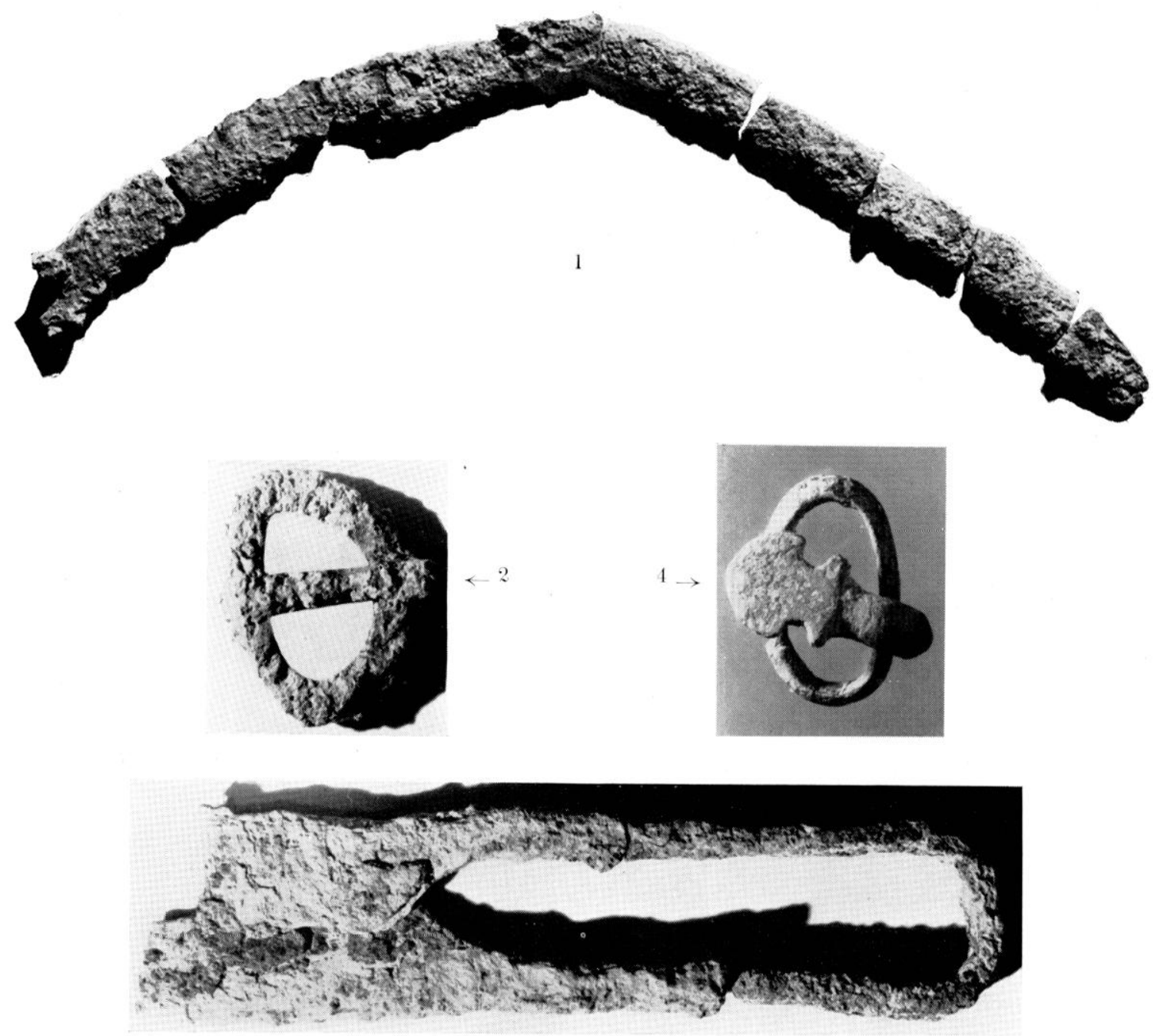

3

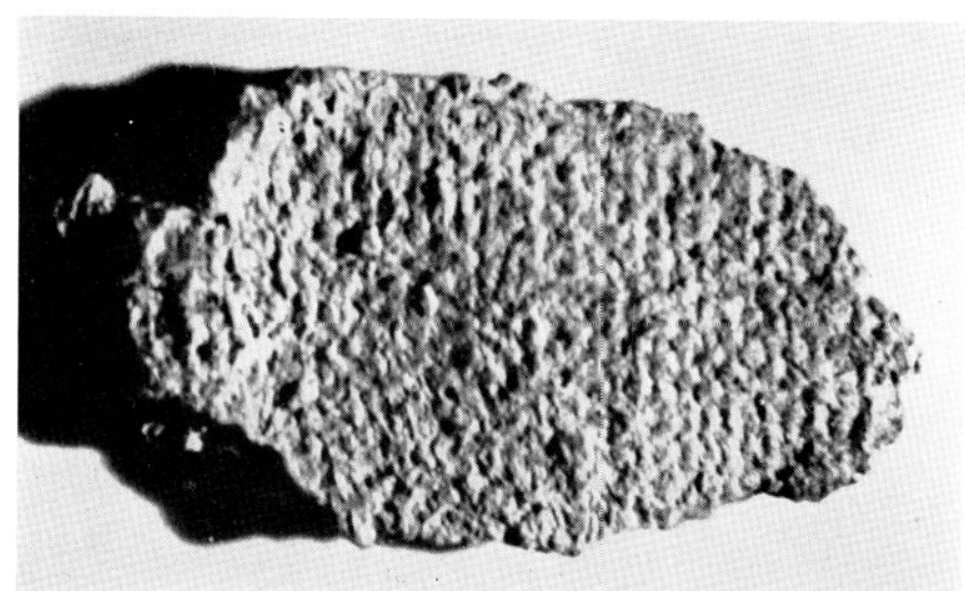

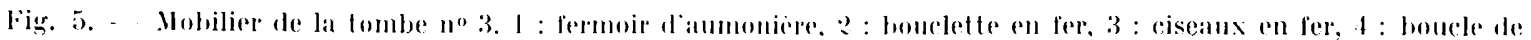

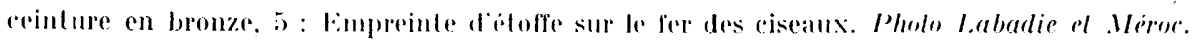




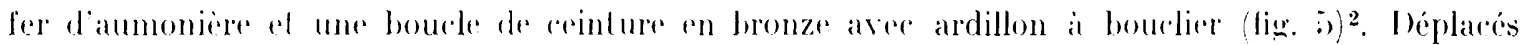

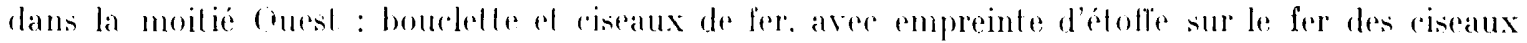

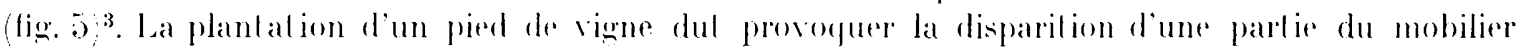
(poinle des riseaus non relrourios).

Tombe t. - Presque entienement démolie par les l ravaux agrieoles antérieurs, il en subsistait sculement une partie de l'extrémite ()uest avec restes d'un raine endommagé.
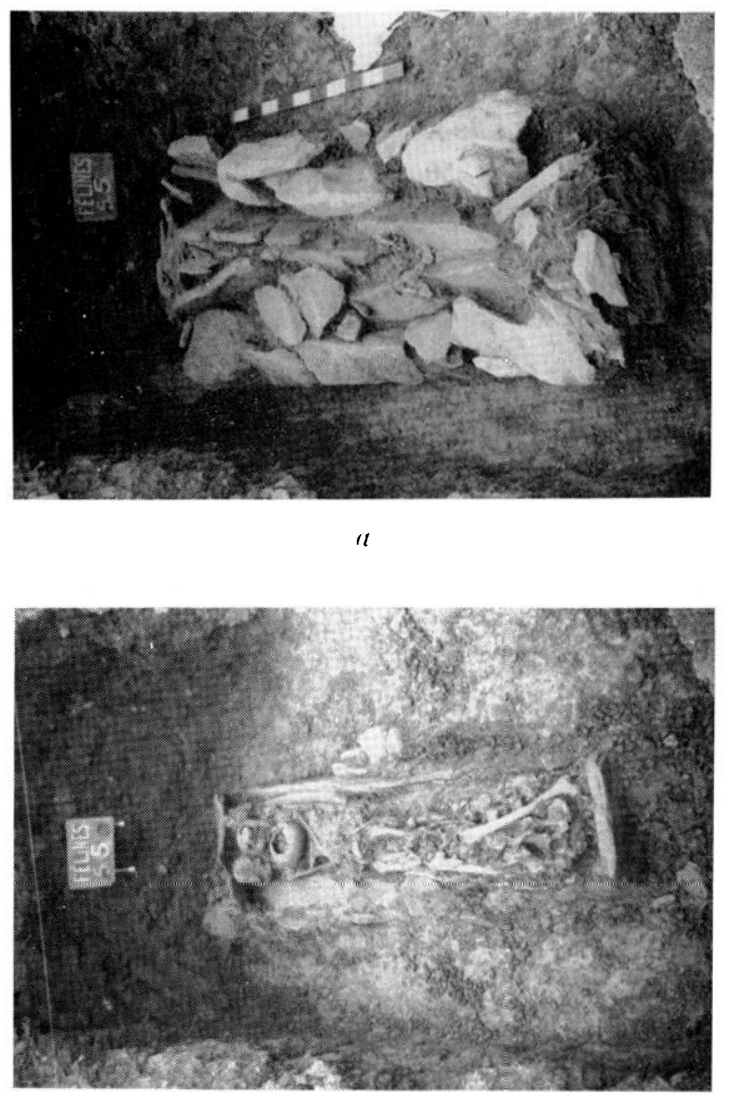

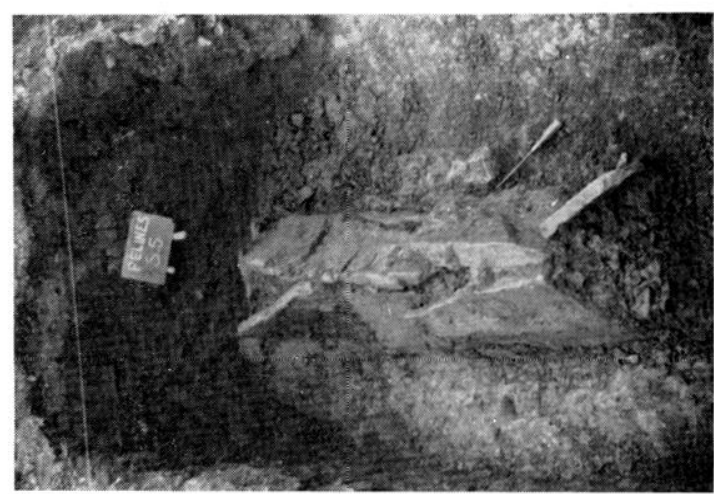

1)

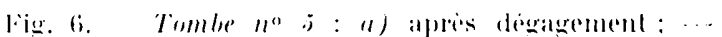
b) avant enlevement des derobieres dalles du toit ; -

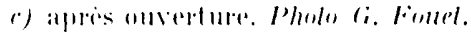

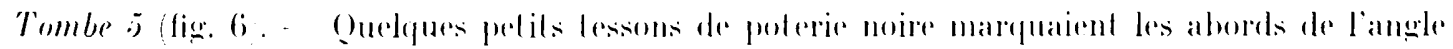
Cord-chest. Des fragments dossements sont apparus dans les interslices du laite de la couverlure: les os eximemes abondaient des le sommet du remplissage terreux intérieur. Ce lombeau rentermait 7 squelelles d'adultes. Ies os de 4 individus au moins, enleves pour la mise an place du dernier defunt dont les mains blaient ramenés sur le bassin avaient, des couverture de lerre du cardavere,

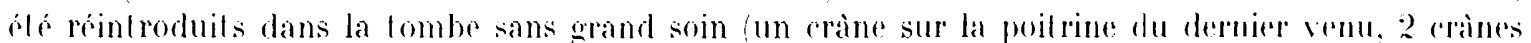
sur sa jambe gauche. les restes des os dissémines au hasard). Ies pelits fragmenls restant en fin d'inhumation avaient foul de meme de rassembles pour etre ajoulés lors de la pose de la couverture

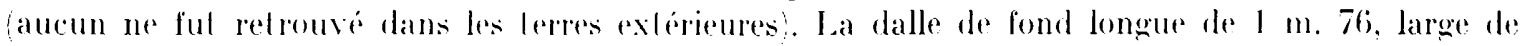

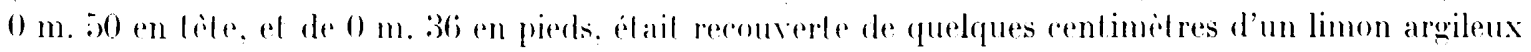
analogue a relui de la lombe no 1 . Aurou mobilier n'y figurail.

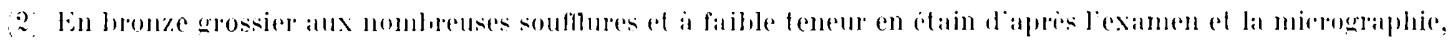

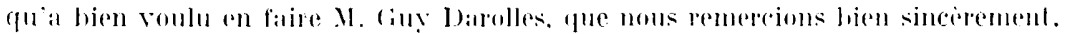

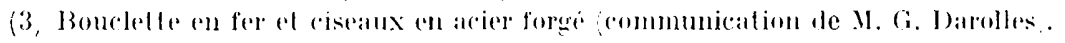


Tombe 6 (lig. 7, . Son fond est formé de deux dalles diune longueur lobale de 1 m. 2i), pour une largeur de $0 \mathrm{~m}$. 38 a $0 \mathrm{~m}$. 28. Trois enfants y avaient éte suceessirement inhumós dont le plus àgé ne devait guere avoir qu'une dizaine d'années. I ne rhape lerreuse les recouvrait. Leurs os. Iress mal conservés n'étaient accompagnés d'aucun mobilier.

Tombe \%. Sa couverture arasée. lémoignail diume violation folale. Sa dalle de fond mesurait

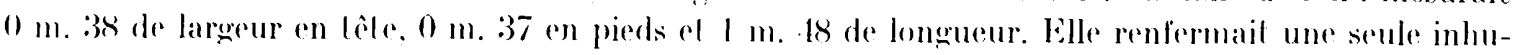

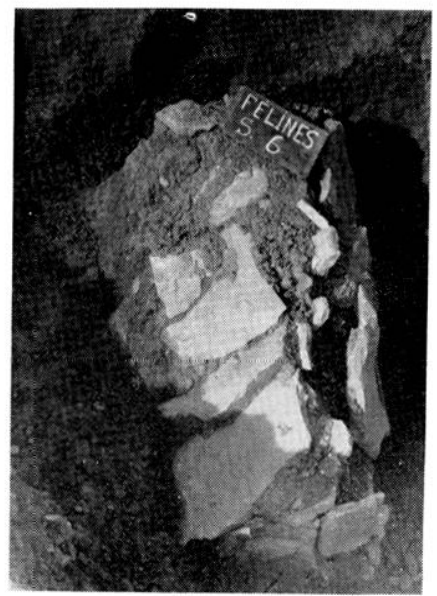

Fin. T. Tombe no b. Vine

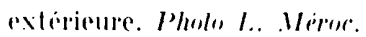

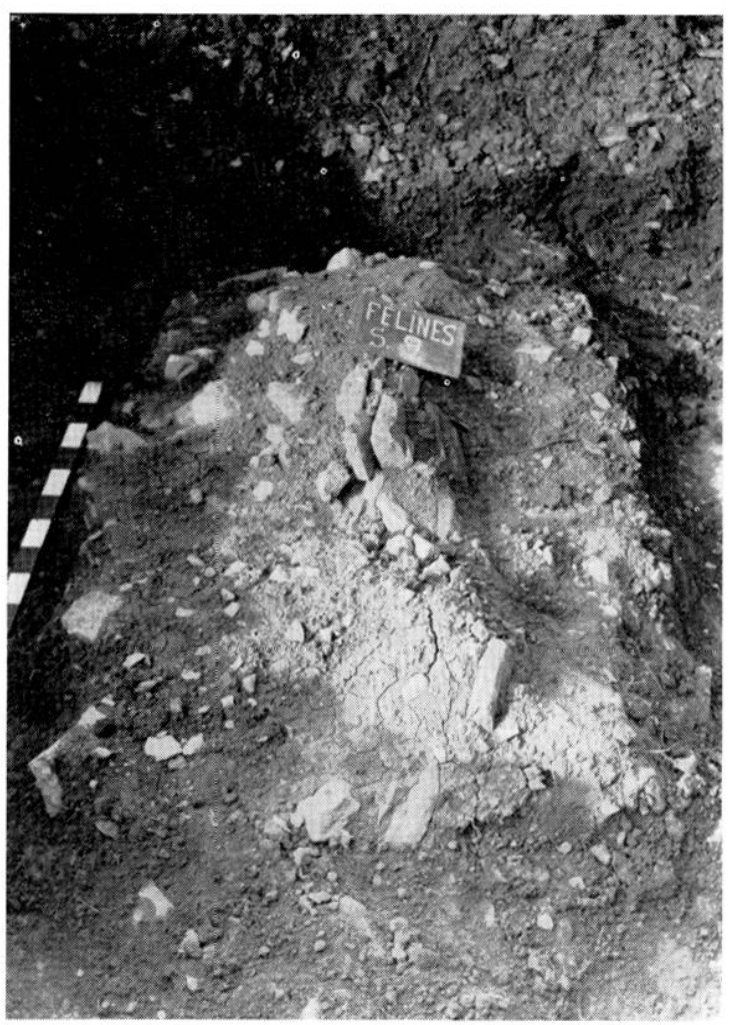

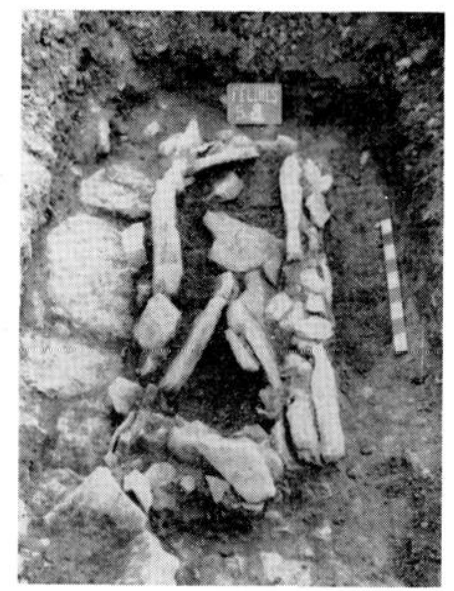

Fix. S. Tombe $n^{\circ}$ s. violien. comme lindique le desorilde des pieries. Pholo Ci. Foltol.

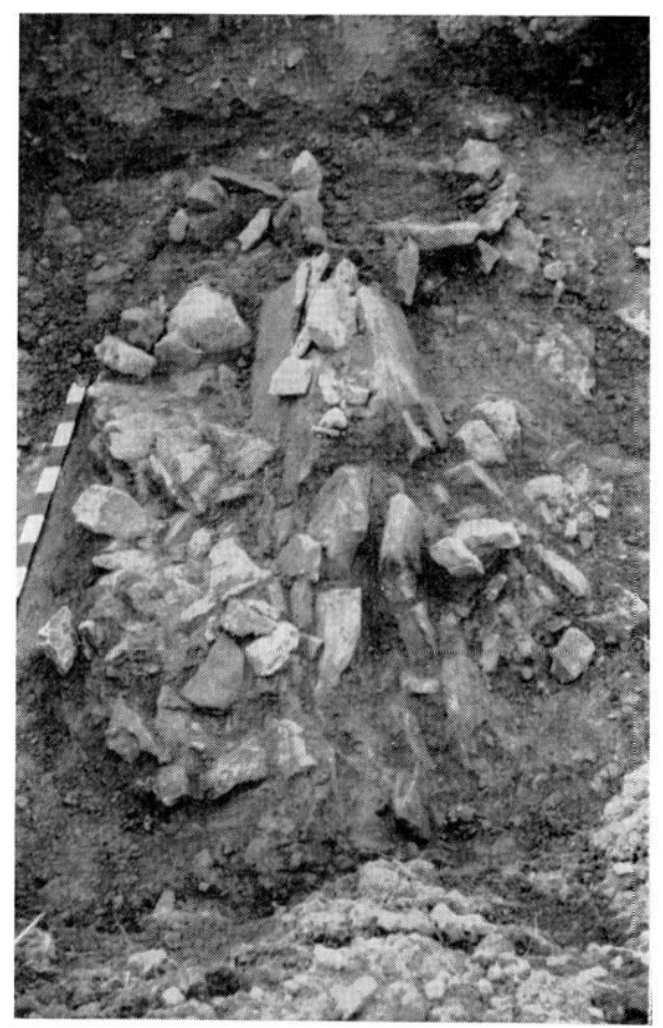

Figr. 9. - Tombe no 9. a deux slades du digagement. Mholes L. Verror al G. Fonel. 

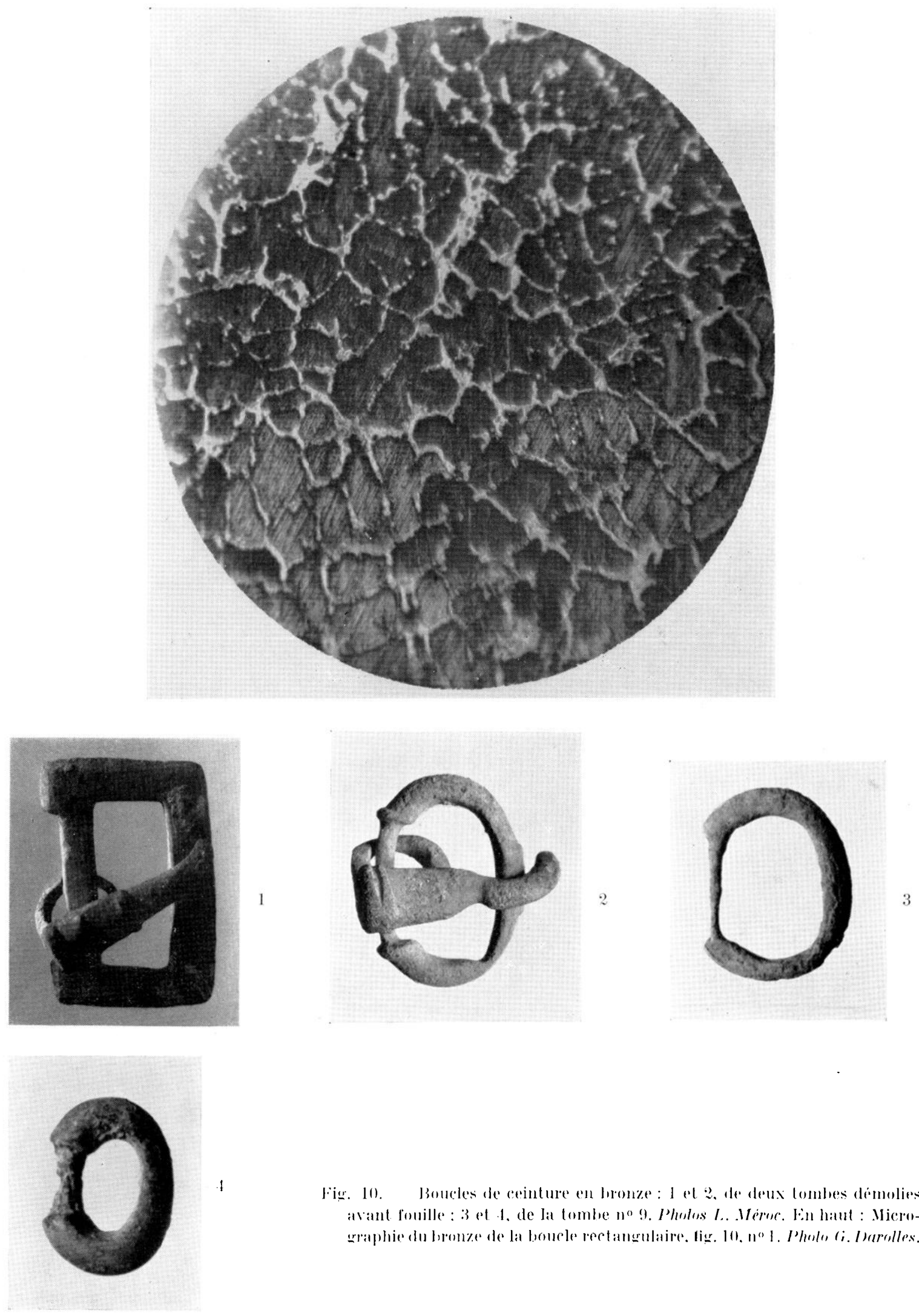

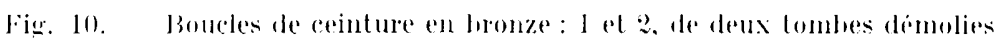

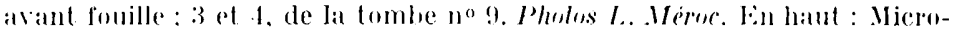

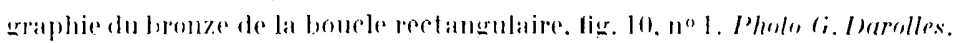


mation : celle d'un enfant d'une dizaine d'années dont subsistail seulement le cràne écrasé sur place. l)ans le remplissage avec des grains de charbon très dispersés présents partout, figuraient 3 menus fragments de briques, 3 morceaux de silex, d'infimes lessons noirs, ol 2 petits escargots d'introduction probablement récente.

Tombe $\&$ (fig. 8). - Sous une couvert ure presque entierement démolie, elle renfermait le squelelte d'un adulte. mais elle avait élé violée, el ne contenait le moindre mobilier. Son fond avait $1 \mathrm{~m} .7 .1$ de longueur sur $0 \mathrm{~m} .10$ de largeur en lite et $0 \mathrm{~m}$. 35) en pieds.

Tombe 9 (fig. 9). - Eille étail remarquable par l'abondante accumulation de fragments de dalles qui formaient chape au-dessus et autour d'une couverture en bâtière assez faible. Les dimensions de son fond étaient de : $1 \mathrm{~m}$. 8i) pour la longueur et de $0 \mathrm{~m}$. (5i) i $0 \mathrm{~m}$. 5) pour la largeur. Elle constiluait la sépulture d'un adulte aux os assez mal conservés. les bras le long du corps mais avec la main droite posée sur la face interne de la cuisse. Deux boucles de bronze (fig. 10) se (rouvaient audesisus des genoux entre les cuisses.

Il semble qu'initialement (au moins dans les tombes nos 1 et (j) les corps aient été simplement déposés sur la dalle de fond préparée à la laille voulue et que les sarcophagres aient été fermés par-dessus aussi hermétiquement que possible. Par la suite les dernières inhumations s'accompagnaient d'apports terreux dans les coflrages b) et 6. Des grains de charbons très dispersés gisaient éparpillés dans les tombes aussi bien que sur leur pourtour mais nulle trace de feu n’a pu être discernée.

Plus du quart des sépultures interrogées renfermaient des inhumations multiples, prohablement familiales : enfants seuls $\left(n^{0} 6\right)$. adultes seuls $\left(n^{0} 5\right)$. jeunes enfants déposés dans la tombe probablement d'une femme (no 3). enfant et adolescents accompagnés de persomnes agées (n $\left.n^{0} 1\right)$. I n liers des inhumés étaient des enfants 4 . Le mobilier funéraire, recueilli uniquement sur quelques adultes, se réduit à de menus accessoires usuels dépendant du costume. Sans doute, les occupants de ce petit cimetière témoignent d'un groupement de quelques familles menant une vie paysanne sédentaire.

Toutes les tombes étaient soigneusement orientées vers le soleil levant (nos 6 et 7 exartement vers l'Est ; légères dérivations vers le Sud-Est pour les autres, de :

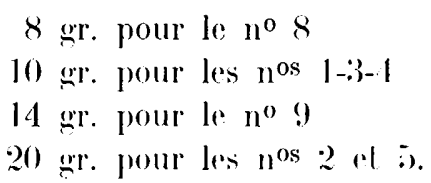

lilles se trouvaient netlement séparés les unes des autres, sans aceolements ni chevaurhements, a qui suppose, lout comme la pratique des inhumations multiples, l'existence de reprères fixes superficiels acturllement détruits par les cultures. L'homogénéilé de l'ensemble ressortant de l'uniformité des tombes semble bien révéler une utilisation de la nécropole durant peu de générations.

Cies coffrages de dalles a converture en bâtière reproduisent manifestement, à l'aide de matériaux de fortune prélevés sur place, la forme des caissons romains à inhumation,

(1) Tous les squelettes en edat comenable de conservation ont etie preleves aux fins d'étude anthropologique. 
de section triangulaire. construits en legulae et imbriess. Les deux types roisinaient i Félines mème: ¿) ou 600 mètres au Sud-bst de Saint-Peyre s'étend un autre cimetière mérovingien sous l'actuel village, entre la route principale et le cours de "l'Ognon ". La parcelle $n^{0} 700$ (jardin de M. Cros) en recouve une partie. Ln caisson intart de briques romaines y avait été exhumé il $y$ a 25 ) ans environ, et c'est au mème endroit (intervalle d'à peine 2 mètres ou 3) que vient d'ètre découvert un coffrage de dalles de forme identique ${ }^{6}$. Plus tardifs que les derniers sareophages romains de section triangulaire en tegulae ou dalles de pierre des $\mathrm{r}^{\mathrm{e}}$ et re siècles dont, le plan reste rectangulaire ${ }^{7}$, les coffrages a hase trapézoïdale de Saint-Peyre ne sauraient probablement ètre antérieurs au début, du vie siècle ${ }^{8}$. Le fait qu'ils restent très proches du type originel, avec espacements réguliers, orientation constante vers le soleil levant, absence d'accolements ou de chevauchements, ferail néanmoins opiner, en faveur d'une date nettement antérieure au vire siècle a a l'égal des indices observables dans te mobilier. Seules, des boucles de petite taillepo dénuées de caracteres tardifsil et que quelques particularites d'ardillons situeraient dans le cours du vie siecte ${ }^{2}$ maintenaient les reintures. Elles s'averent analogues a des trourailles de départements régionaux : Gard ${ }^{13}$, Hérault ${ }^{14}$, Aude ${ }^{15}$, Pyrénées-Orientales ${ }^{16}$. Les épingles restent de type ancien très simple17.

Le site enfin du cimetière appellerait quelques remarques.

Tout près, à une centaine de mètres environ au Nord-Ouest, sur la mème terrasse,

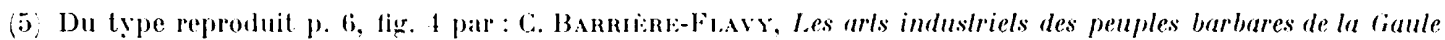
du Ie au VIII siecle. Toulouse-Paris. 1901. I.

(6) Nous devons la comnaissance de ces decourertes a .I. Cros, de biblines à qui nous prosentons de biens amicaux remerciements.

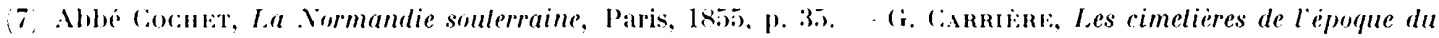
Bas-Empire, de Ponzilhac, Arpaillargues el aulres lien.t du deparlement du Ciard. dans Mémoires Académie de Nimes, XXV, 1902, p. 17-21. - - E. SAl.x, La civilisulion meroringienne, II, Paris, 1952, p. 92-9.1. -..- Sarcophage d'enfant construit en batiere avec des dalles de marbre dans la necropole wisigothique d'Arnesp a Valentine (Haute-(iaronne. Toutes les tombes ou cofirates de ce cimeliere du ve siocle dates extremes 118 -jot sont rectangulaires fonilles inelites 1959 .

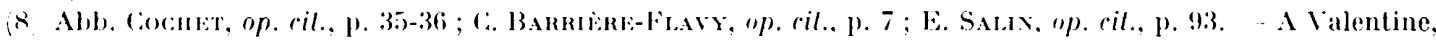
les tombes trapezoidales napparaissent quavec des sarcophages monolithes de calcaire tendre a un niveau supérieur all precedent, et postirieur à loulli.

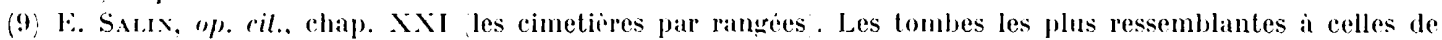

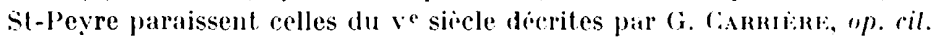

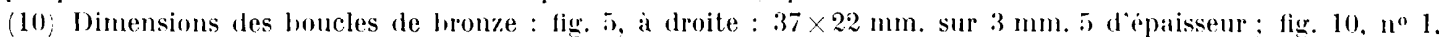

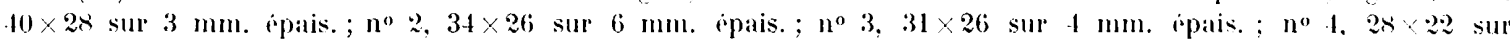
7 min. ipais.

(11) H. ZEIss, Die Cirahfunde aus den spanischen Weslgolerreich, Berlin-I.eipzigr, 1931, p. 81 et planches : ancune des boucles de Fèline n’a les caractires tardifs apparaissant sur les boucles d'ispacrne a partir de jôll.

(12) F. SAIx, op. cil., p. 313 et III, p. 116. Base rectangulaire et boucle d attache coudee tries forte sur les ardillons de Féline.

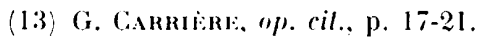

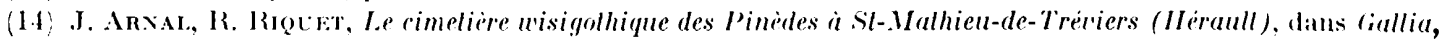
XVII, 1959, 1, p. 161-170, fig. I1.

(15) C. Barritri-Favi, Elude sur les sépultures barbares du Midi el de l'Ouesl de la France, Toulouse-l'aris. 1892, p. 124 et pl. X, no 12 , Armissan .

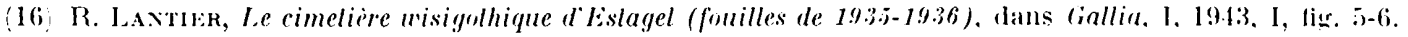

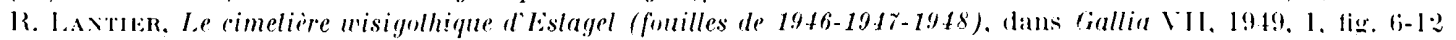
(no 1 : ardillon;.

17, Type gallo-romain present au sve siecle dans la villa de Montmaurin; dans les tombes gallo-romaines de Pouzilhac (G. CAnrti:nt, op. cit., p. 19 ; dans les tombes wisigothiques du ve siecle de lalentine. 
subsistent, sous une vigne, les ruines d'une petite villa romaine. S'ils étaient encore debout, ces murs avaient pu conslituer l'habitat des inhumés de Saint-Peyre. Cethe proximilé explique, en tout cas, la présence des menus morceaux de brique et tessons retrouvés.

D'autre part, de nombreux fragments de lames, pointes de flèche, et éclats de silex parsèment la surface du cimetière (surface hachurée, fig. 1). Ces vestiges d'une petite station néolithique peuvent avoir suggéré le choix de l'emplacement, les gens de l'époque mérovingienne ayant, souvent accordé pouvoir tulélaire aux silex qu'ils déposaient parfois, comme phylactères, dans les sépultures ${ }^{18}$.

Le vieux chemin bordant, Saint-Peyre et menant, vers l'église actuelle paraît être par ailleurs l'ancienne route qui, venant de Trausse, monlait par Camplong vers SaintJulien-des-Molières sur le Causse.

En attendant des données complémentaires en ces divers lieux ${ }^{19}$, d'utiles comparaisons peuvent déjà s'établir entre les observations faites d'une part sur les sépultures en coffres plats en forme de cercueil de Saint-Mathieu-de-Tréviers et surtout d'Estagel ${ }^{20}$, et d'autre part sur les tombes en bâtière de Saint-Peyre, où apparaît la survivance des tradilions gallo-romaines parmi les populations rurales des bordures du Causse.

I. Mńroc, (i. Fotet.

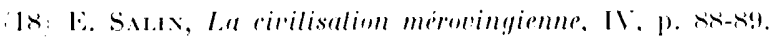

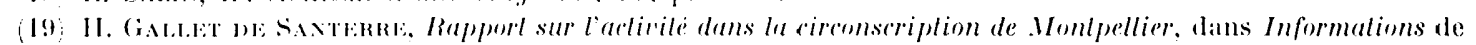
(iallia. XVII, 1959, 2. p. 469 . Le ban de Febines se revile riche en cimelieres merovingiens qu'il serait instructif d'itu-

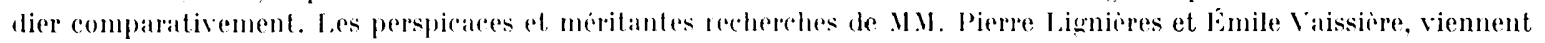
deja d'en décour rir six. Trois sont ceux de hameanx sur des teprasses aux flancs du Causse : Ventajou, Camplongr, Ayquhouno. Mais trois autres apparaissent fort rapproches dans la vallee. An cimetiere signale sous le jardin de M. Cros

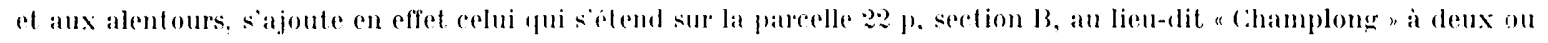
trois centaines de mètres sentement de st-perare dans la meme situation que ce dernier par rapport an verux chemin. (20) M. Iaxtren, diti plus haut. p. 16 . 